\title{
El Desarrollo de los Procesos Sustantivos de la Educación Superior Ecuatoriana ante el Reto de la Inclusión Educativa
}

\section{The Development of the Main Processes of Ecuadorian Higher Education Facing the Challenge of Educational Inclusion}

Rafael Félix Bell *

Instituto Tecnológico Superior de Formación Profesional, Administrativa y Comercial

\begin{abstract}
El análisis y la ejemplificación de algunas de las principales posibilidades que la inclusión educativa tiene para potenciar el desarrollo de los procesos sustantivos en torno a los cuales se articula la labor de las Instituciones de Educación Superior, constituyó la principal motivación para la realización de la investigación en cuyos resultados se sustentan las reflexiones y conclusiones del presente artículo. En el mismo se destaca el papel de los docentes, del sistema de acompañamiento metodológico y de la participación protagónica del estudiantado para la transformación del aula en un ambiente de aprendizaje en el que todos puedan encontrar los apoyos requeridos y se les garantice una respuesta educativa de calidad. De igual manera se resaltan las contribuciones de la inclusión educativa para la investigación, la gestión y para el reforzamiento del carácter doblemente inclusivo de la vinculación.
\end{abstract}

Descriptores: Enseñanza superior, Docencia, Aprendizaje, Investigación, Gestión.

Analysis and exemplification of some of the main possibilities that educational inclusion is to enhance the development of substantive processes around which the work of the Institutions of Higher Education is articulated, was the main motivation for the development of research in the results of the reflections and conclusions of this article are based. This paper highlight the role of teachers, the system of methodological support and active participation of students to transform the classroom into a learning environment in which everyone can find the support required and guaranteed an education response stands quality. As well as, the contributions of educational inclusion for research, management and for strengthening the doubly inclusive bonding are highlighted.

Keywords: Higher education, Teaching, Learning, Research, Management. 


\section{Introducción}

La inclusión educativa representa uno de los principales desafíos para las Instituciones de Educación Superior en los momentos actuales y demanda la integración e intensificación de las acciones con el fin de lograr que las aspiraciones y propósitos reflejados en leyes, declaraciones y lineamientos se hagan realidad en la práctica educativa.

En ese empeño, múltiples son los obstáculos que deben ser superados, pues la creciente presencia de estudiantes con las más diversas características en entornos de aprendizaje de la Educación Superior no siempre encuentra la respuesta requerida en el nivel de preparación del profesorado y del propio estudiantado con relación a los métodos, formas de organización e interacción y herramientas para poder asumir de manera exitosa el reto de la diversidad y por tanto, el cumplimiento de los objetivos del proceso de inclusión.

Los esfuerzos que en el Ecuador se despliegan en la dirección indicada y de manera más específica los que tienen a la población estudiantil con alguna discapacidad como uno de sus centros de atención, resultan coherentes con los preceptos refrendados en la Constitución de la República del Ecuador (2008), con los principios de la Ley Orgánica de Educación Superior (2010), con los objetivos del Plan Nacional del Buen Vivir (20132017) y con los enfoques de las declaraciones y convenciones internacionales de mayor relevancia en esta materia, en particular la Declaración de Salamanca (1994) y la Convención Internacional de las Naciones Unidas sobre los Derechos de las personas con discapacidad (2006).

En su art. 71, por ejemplo, la Ley Orgánica de Educación Superior, consagra el principio de igualdad de oportunidades que consiste en "garantizar a todos los actores del Sistema de Educación Superior las mismas posibilidades en el acceso, la permanencia, movilidad y egreso del sistema, sin discriminación de género, credo, orientación sexual, etnia, cultura, preferencia política, condición socioeconómica o discapacidad" (CES, 2013, p. 79).

La letra y el espíritu de los documentos citados brindan un sólido soporte a la Fundamentación y lineamientos para transversalizar los ejes de igualdad y ambiente, que bajo el título "Construyendo igualdad en la Educación Superior" (2015) fueron publicados por la Secretaría de Educación Superior, Ciencia, Tecnología e Innovación (SENESCYT) del Ecuador con el siguiente propósito:

Fortalecer los procesos de construcción de la igualdad en y desde la educación
superior, teniendo como fin último el compromiso por la conformación de una
sociedad igualitaria, democrática e incluyente y el reconocimiento del rol de
liderazgo que el sistema de educación superior está llamado a ejercer en la
transformación de las prácticas sociales. (Herdoiza, 2015, p.11)

De esta manera, las IES se han de colocar en una posición de vanguardia que les permita incrementar su aporte en los debates conceptuales que hoy tienen lugar en torno a la inclusión y sobre todo, en la elaboración y aplicación de propuestas para su exitosa introducción y generalización, con los ajustes que puedan ser requeridos, en la práctica educativa.

Al respecto, cabe resaltar la naturaleza compleja que encierra el concepto de inclusión educativa, lo que provoca que surjan confusiones acerca de su contenido (Echeita y Ainscow, 2011) que, a la vez, son una evidencia de su carácter abierto y de su propia 
construcción, en particular a nivel de la Educación Superior, en cuyo ámbito todavía constituye un joven proceso en pleno desarrollo.

No hay que obviar que se trata de un concepto que surgió en el seno de la denominada Educación Especial, luego transitó por el modelo conocido como integración escolar que constituye el antecedente inmediato de la inclusión educativa, que es el escalón más alto de este recorrido, como se refleja en el siguiente esquema:

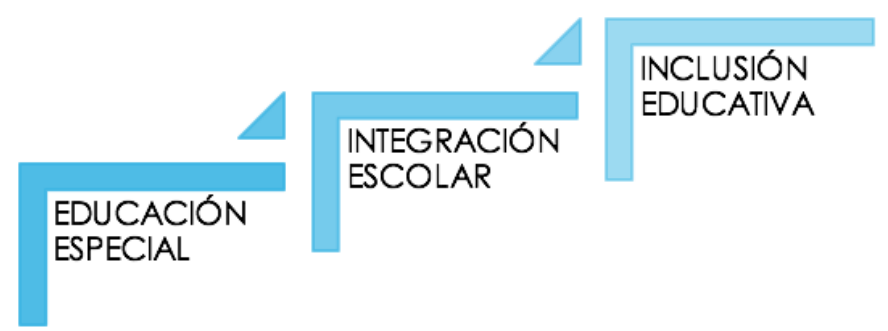

Figura 1. Evolución de la Educación especial a la Inclusión educativa Fuente: Elaboración propia.

En consonancia con lo antes expuesto y reconociendo las potencialidades de la inclusión educativa para favorecer las transformaciones que la Educación Superior requiere, la hipótesis general de la investigación en la que se fundamenta el presente artículo se formuló de la siguiente manera: la inclusión educativa potencia el desarrollo de los procesos sustantivos de la Educación Superior. Esta hipótesis fue verificada en el entorno de aprendizaje del Instituto Tecnológico Superior de Formación Profesional, Administrativa y Comercial, una IES acreditada de la ciudad de Guayaquil, Ecuador.

Consiguientemente, a partir de la comprensión de la naturaleza compleja de la inclusión educativa, el objetivo de este artículo es analizar algunas de las principales implicaciones que este proceso tiene para la docencia, la investigación, la vinculación y la gestión, funciones que definen la esencia de la labor de las IES y revelan, al mismo tiempo, su pertinencia social.

\section{Fundamentación teórica}

\subsection{Docencia, trabajo metodológico e inclusión}

La docencia constituye el eje vertebrador de la actividad de las IES. Sin embargo, los modelos formativos que hoy se aplican parecen no considerar suficientemente los significativos cambios que continúan teniendo lugar en la sociedad como resultado, entre otros factores, de la globalización y del incesante desarrollo de las Tecnologías de la Información y de las Comunicaciones, que demandan una profunda transformación de la docencia y en especial, del papel del profesor universitario.

Según Montenegro y Fuentealba (2010), basados en el informe OCDE (2009), en las aulas universitarias prevalece la aplicación de métodos tradicionales de enseñanza, que se caracterizan por el énfasis que colocan en el aprendizaje individual, la marcada orientación hacia la memorización y por una evaluación homogénea y eminentemente reproductiva. En el caso de la evaluación, por ejemplo, el diseño de varios tipos de pruebas, cuando se realiza, responde más al interés de los docentes por evitar que los 
alumnos se copien, que por elaborar instrumentos de evaluación que respondan a la diversidad presente en el aula.

En ese contexto y como expresión del predominio de la competitividad en las aulas, de la estructuración atomizada del currículum, de la rigidez en la organización espacio temporal de la actividad docente (López Melero, 2011) y de una arraigada visión del profesor universitario como transmisor de conocimientos, se generan barreras didácticas, que al igual que las barreras arquitectónicas, psicológicas, tecnológicas y culturales, se convierten en obstáculos que limitan y en ocasiones impiden el cumplimento de los objetivos de la inclusión.

A los aspectos señalados convendría añadir la escasa consideración de la caracterización de los estudiantes, que no permite establecer con claridad su nivel de partida (Talízina, 1993) para asumir los desafíos que el proceso de enseñanza-aprendizaje presupone. El establecimiento y la consideración del referido nivel de partida favorecerían la superación de las consecuencias que conlleva la aplicación de un modelo didáctico orientado a las necesidades y potencialidades de un supuesto alumno promedio, lo que dificulta el ajuste de la respuesta educativa a la diversidad del estudiantado.

Como reflejo de la referida dificultad, en no pocas ocasiones la reacción inicial de los docentes de la Educación Superior ante la presencia en el aula de estudiantes con capacidades especiales se evidencia en el cuestionamiento de ese hecho, de las razones y vías que lo han propiciado y a veces se llega a abogar por la conveniencia de la incorporación de esos estudiantes a otros espacios para su formación tecnológica y profesional superior. Esa situación genera la sensación de un nuevo intento por regresar a la Educación Especial pero esta vez a nivel universitario.

Todo ello constituye una clara manifestación del largo camino que queda por recorrer en las IES para el logro de los propósitos de la inclusión educativa, en cuyo recorrido no hay que obviar que el ajuste de la respuesta educativa no ha de interpretarse como un hecho aislado, como la tarea exclusiva de un profesor por separado, pues la superación de las distintas barreras que obstaculizan la inclusión y en específico las de tipo didáctico, requiere la conjugación del esfuerzo individual de cada profesor con la aplicación de diversas acciones y estrategias colectivas para elevar la calidad de la docencia en el Instituto, entre las que se destaca el establecimiento del sistema de acompañamiento metodológico (SAM).

Este sistema presupone la estimulación y el fortalecimiento de la auto-preparación de los docentes, el funcionamiento de colectivos académicos de carreras y materias, los despachos técnicos con el Departamento de Bienestar Estudiantil para el intercambio de información y la precisión de acciones para la atención a los estudiantes, incluidos los que presentan alguna discapacidad. Todo ello se potencia con la observación docente interprofesional con el consecuente análisis de las clases, que en ocasiones puede ser realizada mediante la grabación, observación y análisis de videos de fragmentos de actividades impartidas por el propio docente y por otros profesores.

Así, el SAM constituye una apuesta para el logro de la maestría pedagógica del profesorado, lo que repercutirá positivamente en la elevación de la calidad de las clases y por consiguiente, en la formación de tecnólogos con una mejor preparación profesional e integral. Acerca de este último aspecto conviene resaltar que, en este caso, la imprescindible orientación educativa de las acciones metodológicas que se desarrollan en 
la Educación Superior se refuerza con la introducción de la figura del profesor tutor integral, que es asumida por un docente que propicia la interacción sistemática con un grupo de estudiantes y se convierte en un catalizador de la acción educativa y de la atención que puedan requerir los inconvenientes e inquietudes individuales y colectivos, dentro de los cuales se le ha de prestar una especial atención a los vinculados con la inclusión educativa.

Las acciones y estrategias del SAM incorporan importantes elementos para la innovación del desempeño profesional de los docentes y se convierten, como ha señalado Ainscow (2004) en palancas que han de favorecer el cambio, teniendo como premisas la reflexión y la investigación de los formadores acerca de su propia práctica. Se generan de este modo condiciones para la transformación, que es una de las esencias de la innovación, que si es auténtica, no se ha de limitar al terreno tecnológico sino que ha de penetrar la esfera de la comunicación (Del Castillo et al., 2013) y toda la cultura de las IES, orientándolas hacia el aprendizaje y la activa transferencia del conocimiento, utilizando para ello diferentes mecanismos y estimulando la creatividad a fin de superar las formas y contenidos tradicionales del intercambio académico, que con frecuencia se produce en espacios y circunstancias que no siempre garantizan la potenciación de su impacto innovador.

Al compartir necesidades y anhelos comunes, la puesta en marcha del SAM encuentra múltiples puntos de contacto con experiencias como la referida al trabajo metodológico (Tristá y Álvarez Vázquez, 2010), la relacionada con el desarrollo del saber académico sobre la enseñanza (Montenegro y Fuentealba, 2010) y también con el denominado Lesson Study (Cerbin y Kopp, 2006), que en esencia reflejan la inquietud y el interés de los profesores e investigadores de la Educación Superior por construir de manera conjunta un conocimiento pedagógico y didáctico que favorezca el mejoramiento de la docencia en respuesta a las exigencias de la sociedad actual. Todos estos empeños confirman que "una de las principales innovaciones educativas es la creación de equipos docentes. Quizá sea de una de las más necesarias” (Michavila, 2009, p. 6).

En esa dirección se hace indispensable promover la protagónica participación de los estudiantes, que ha de ser potenciada mediante la asignación de tareas y responsabilidades como parte de los equipos para el aprendizaje cooperativo, la conformación de dúos o pares para el apoyo mutuo en la resolución de problemas o tareas y el aprovechamiento de las opciones que ofrece la intervención de los estudiantes seleccionados como ayudantes de cátedra e investigación.

La incorporación de estudiantes con capacidades especiales a la ayudantía de cátedra e investigación abre nuevos horizontes para la realización de sus prácticas pre profesionales, que les brindan la posibilidad de cumplir con las diferentes funciones y responsabilidades previstas en el Reglamento de Régimen Académico y, a su vez, contribuyen a revelar una de las aristas del verdadero carácter inclusivo de una IES.

Al hilo de lo hasta aquí señalado es evidente la importancia de garantizar el establecimiento y adecuado funcionamiento de un sistema de acompañamiento metodológico como condición para el logro de niveles de excelencia en la docencia de la Educación Superior, que tiene en el cumplimiento de los objetivos de la inclusión educativa un compromiso impostergable y una fuente para la innovación y el crecimiento humano y profesional. 


\subsection{La investigación ante el desafío de la inclusión}

En el literal c del artículo 5 de la Declaración Mundial sobre la Educación Superior para el siglo XXI (UNESCO, 1998) se subraya que "cuando la educación superior y la investigación se llevan a cabo en un alto nivel dentro de la misma institución se logra una potenciación mutua de la calidad" (p. 24).

Por consiguiente, es creciente y justificado el interés por la investigación en las IES, una de cuyas prioridades es la orientación hacia la solución de necesidades y problemas de la realidad socio-económica, cuya comprensión y análisis no serían completos si no se incorporan y abordan con sensibilidad humana y rigor científico los complejos temas de la inclusión educativa y social.

Sin embargo, los referidos temas no siempre son considerados en los diseños de los planes de investigación y con frecuencia, no se aprovecha ni se promueve la participación de los estudiantes con discapacidad en la investigación de situaciones y realidades de las cuales ellos son protagonistas y cuentan con vivencias y experiencias que mucho podrían aportar al estudio de la educación de las personas con discapacidad y de la diversidad en general. Por consiguiente:

La inclusión educativa no puede separarse del concepto de voz y que, por lo tanto, los
dilemas sobre nuestra docencia y nuestra investigación han de llevar consigo
necesariamente una reflexión y un compromiso con la democratización y la
afirmación del derecho de las personas o grupos habitualmente silenciados a ser
escuchados. (Susinos y Rodríguez-Hoyos, 2011, p. 17)

No obstante, quedan obstáculos por superar y para ello será necesario promover el aprovechamiento de las posibilidades de la investigación para avanzar, entre otros temas, en la intensificación de la aplicación de los métodos activos para el aprendizaje, en la optimización del uso de las TIC como un poderoso recurso de apoyo y dinamización del proceso de enseñanza-aprendizaje, en la diversificación de las opciones para la evaluación y en el perfeccionamiento de las vías para la atención de las necesidades individuales de cada estudiante. Todo ello se encuentra estrechamente relacionado con la preparación del profesorado, con su actividad en el aula en interacción con sus estudiantes y en la acción tutorial, que constituyen importantes pilares para la inclusión educativa en las IES.

\subsection{Vinculación con la sociedad}

La introducción del término vinculación puede considerarse como un hecho relativamente reciente en el entorno de la Educación Superior, en el que, con anterioridad, era habitual identificar esta función bajo denominaciones como extensión o difusión. No obstante, conviene precisar que el asunto no se limita a una cuestión terminológica, pues encierra, ante todo, una posición conceptual, que como ha precisado Gould (2001) citado por Alcántar y Arcos (2004) pretende subrayar la naturaleza educativa, científico-tecnológica, social y humana de este importante proceso.

En esencia la vinculación puede ser definida de la siguiente manera:

Conjunto de procesos y prácticas planeados, sistematizados y continuamente evaluados, donde los elementos académicos y administrativos de una IES se relacionan externamente con otras personas y organizaciones, con el propósito de desarrollar y realizar acciones y proyectos de beneficio mutuo. (Machucho y López, 2012, p. 12) 
Por tanto, la vinculación permite el establecimiento de puentes entre las IES y diversos sectores sociales, productivos y de servicios, contribuyendo de esa manera a la promoción de la pertinencia institucional, al reconocimiento de las IES en la sociedad, al mejoramiento de su imagen y a un mejor posicionamiento en su radio de acción (Alcántar y Arcos, 2004).

En línea con el objetivo del presente artículo cabe resaltar el carácter humano y social de la vinculación que, como apunta Malagón (2006) está determinado por el propósito de garantizar, junto con el desarrollo de las competencias científico-técnicas, la formación integral de los futuros profesionales, quienes han de actuar como catalizadores de las transformaciones requeridas para el mejoramiento de la calidad de vida de toda la población, en particular, de quienes proceden de sectores sociales cuya atención constituye una prioridad.

En ello se expresa la dimensión inclusiva de la vinculación, que alcanza una trascendencia todavía mayor cuando se logra que en sus diversas actividades y proyectos participen activamente todos los estudiantes, algunos de los cuales, como los estudiantes con discapacidad, pertenecen a los sectores que demandan una priorizada atención. Se revela así la naturaleza doblemente inclusiva de la vinculación, derivada de la interacción entre quienes participan en el diseño y conducción de las actividades y aquellos que se ven beneficiados directamente con su realización.

Esa interacción se ve favorecida por las características de los ambientes de aprendizaje que la vinculación genera, en los que impera la flexibilidad, el diálogo de saberes, la confianza y el respeto mutuo. En esas condiciones, los estudiantes, incluidos aquellos que presentan alguna discapacidad, manifiestan de una manera más natural todas sus potencialidades, experimentan la satisfacción de comprobar la utilidad de los aprendizajes adquiridos y acceden a nuevas experiencias, conocimientos y realidades que los estimulan para nuevos aprendizajes.

Al mismo tiempo la participación en las actividades de vinculación de los estudiantes con discapacidad junto con sus compañeros y profesores, representa una importante contribución de la educación superior al desarrollo de prácticas inclusivas (García y Cotrina, 2012) pues sirven como ejemplo vivo y real de esas prácticas y actúan como un efectivo medio para su difusión.

\subsection{Gestión e inclusión}

La gestión puede ser definida como "el proceso en general que comprende cuatro funciones típicas: el planeamiento, la organización, el liderazgo y la evaluación” (Fainholc, 2006, p. 3). Precisamente la función referida al liderazgo es tomada como núcleo de la definición que propone Cantón (1997) citada por Botero (2009), quien señala que la gestión se relaciona con el desarrollo de las acciones que realiza un grupo humano a partir de la orientación de un líder.

La aplicación de estos y otros conceptos provenientes del área de la Administración al ámbito de la Educación Superior constituye uno de los temas que continúa en el centro de atención de expertos e investigadores en esta materia quienes, con frecuencia, subrayan que una de las particularidades de la gestión educativa radica en el reconocimiento del papel que en la misma juegan las competencias humanas (Botero, 2009). 
Esta postura encuentra un claro reflejo en las reflexiones de Sañudo (2005), en cuya comprensión la gestión educativa es definida de la manera siguiente:

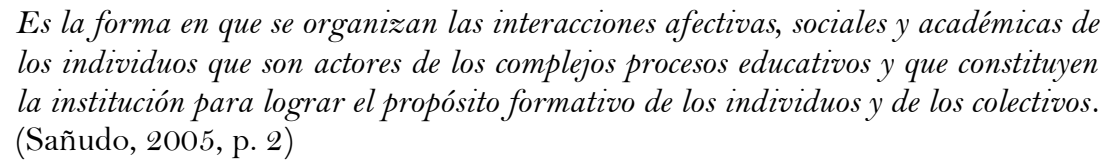

Es la forma en que se organizan las interacciones afectivas, sociales y académicas de los individuos que son actores de los complejos procesos educativos y que constituyen la institución para lograr el propósito formativo de los individuos y de los colectivos. (Sañudo, 2005, p. 2)

Precisamente ese componente humano, de interacción entre las personas, es una de las esferas en las que la inclusión educativa encierra mayores posibilidades para potenciar la gestión de las IES, pues la presencia de estudiantes y profesores con diversas características, incluidas las de aquellos que presentan alguna discapacidad, genera no solo la necesidad de redoblar los esfuerzos en la superación de distintos tipos de barreras, sino de trabajar en la creación y consolidación de una cultura organizacional realmente abierta a la diversidad.

De hecho no son muy abundantes las referencias a experiencias relacionadas con la labor de profesores con discapacidad en las IES. Sin embargo, esas experiencias existen y su registro aporta una valiosa información para la gestión y la actividad en general de la Institución. La interacción con docentes con esas características favorece la manifestación de valores como la solidaridad y el respeto, y ofrece, al mismo tiempo, la posibilidad para la expresión de sus capacidades, que les permiten contribuir al cumplimiento de los objetivos y metas personales e institucionales.

Se constatan, de esta manera, las repercusiones de la inclusión vinculada con el desempeño docente y su impacto en el proceso educativo que tiende a reforzar su influencia cuando la docencia es asumida por un profesor con determinada discapacidad, que se convierte en modelo de esfuerzo, dedicación y perseverancia para sus estudiantes.

En la estimulación y consolidación de la referida influencia y en el avance de las IES hacia la materialización de los principios de la inclusión educativa, a los líderes les corresponde jugar un decisivo papel, que presupone desarrollar, de acuerdo con la teoría del liderazgo transformacional,

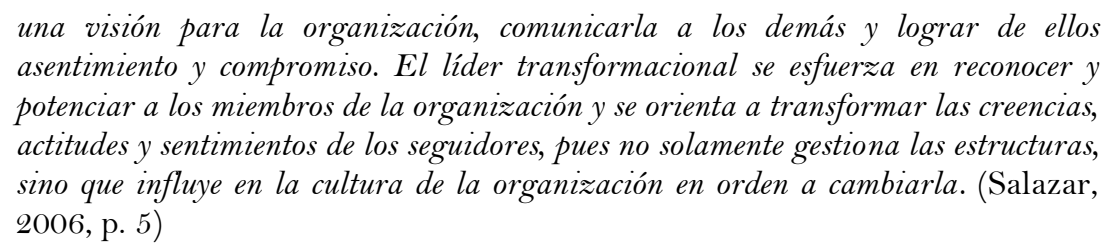

Derivado de ello es posible subrayar que la inclusión educativa puede aspirar a su exitoso desarrollo en las IES si la misma es asumida como expresión de los valores y las creencias de sus líderes, sobre cuya base han de ser capaces de concientizar, motivar y comprometer a sus colectivos para convertir en realidad las altas metas de tan noble y humano proceso.

\section{Metodología}

La investigación de tipo descriptivo-explicativo que sirve de sustento al presente artículo se realizó durante un año, en el período comprendido entre agosto de 2015 y agosto de 2016 y se apoyó en la utilización de métodos teóricos y empíricos, buscando, en el caso de estos últimos, la necesaria complementariedad para una mejor 
aproximación al complejo objeto de estudio que representa la relación entre la inclusión educativa y los procesos sustantivos de la educación superior ecuatoriana.

En cuanto a los métodos de nivel teórico, a partir del empleo de la lectura crítica e inferencial, se recurrió al análisis y la síntesis para el estudio de variadas fuentes de información, entre las que se incluyeron libros físicos y digitales y revistas impresas y electrónicas. Fueron consultados autores de distintos países, cuyos aportes permitieron constatar la existencia de diferentes matices en el abordaje de la inclusión educativa y la manera en la que la misma comienza a ser aplicada en la Educación Superior.

Se requirió también el empleo del método histórico-lógico con el propósito de analizar la trayectoria y evolución del concepto de inclusión educativa y la lógica que ha primado en la configuración de sus enfoques actuales. En esa dirección, la investigación profundizó en la comprensión actual de la inclusión educativa, en su alcance para toda la diversidad del estudiantado y en sus implicaciones para la labor del profesorado.

En relación con los métodos empíricos fueron utilizados la observación, la encuesta en forma de cuestionario y el estudio biográfico narrativo. La aplicación de la observación permitió describir la dinámica de las clases y de las interacciones que en las mismas se establecen entre todos los estudiantes, incluidos aquellos que presentan alguna discapacidad.

Mediante la aplicación del cuestionario y de la observación, se realizó la caracterización que permitió establecer el nivel de partida de los aspirantes a cursar estudios de nivel tecnológico superior en el Instituto Superior de Formación Profesional, Administrativa y Comercial, de Guayaquil, Ecuador.

De igual manera se identificaron, a través de una encuesta realizada con la utilización de la aplicación de Google Forms, las principales características del profesorado, incluidas sus necesidades de formación para una mejor atención a la diversidad.

Por su parte, el empleo del estudio biográfico narrativo, posibilitó un mejor acceso a la comprensión de las vivencias y experiencias de estudiantes, graduados, docentes, colaboradores y familiares en relación con la inclusión educativa, lo que favoreció la obtención de valiosas evidencias acerca de los recursos, estrategias y alternativas empleadas en la búsqueda de una mejor respuesta educativa a la diversidad del estudiantado en la educación tecnológica superior.

Consecuentemente, la aplicación del estudio biográfico narrativo en la investigación desarrollada, se convirtió en una de las vías para la amplificación de las voces de los estudiantes y sus familias, quienes, además de narrar sus historias de vida y experiencias, expresaron inquietudes y trasladaron propuestas dirigidas a continuar mejorando la calidad de la educación que la institución brinda. De esta forma, los métodos aplicados permitieron constatar, como ha sido señalado por Bolívar (2002) no sólo los aspectos formales de la investigación, sino las diversas manifestaciones emocionales, las aspiraciones y frustraciones de sus protagonistas, entre los que sobresalen los estudiantes, los graduados y el docente con capacidades especiales.

\section{Resultados}

El abordaje de los procesos sustantivos de la Educación Superior no puede ser realizado al margen de sus componentes personales más activos, es decir, los estudiantes y los 
docentes. Por tal motivo el establecimiento del nivel de partida de los estudiantes de nuevo ingreso, constituyó uno de los temas de mayor interés de la investigación y la aplicación de los diversos instrumentos de recogida de información a los 128 aspirantes del curso de nivelación, permitió resaltar la presencia, entre otros, de los siguientes factores:

- Las situaciones laborales y económicas que enfrentan los estudiantes y sus posibles repercusiones en la continuidad de sus estudios superiores.

- Las dificultades en el uso del lenguaje, tanto en forma oral como escrita, agravadas por problemas ortográficos y en la comprensión lectora.

- La correspondencia del perfil vocacional con las principales áreas de interés profesional.

- El hallazgo de buenas relaciones interpersonales unidas a la capacidad para mantener el control interno y un adecuado autoconocimiento.

- La diversidad de capacidades, edades y de experiencias educativas, culturales, familiares y sociales de los nuevos estudiantes.

Ante la realidad constatada, resultó de gran valor propiciar un mejor conocimiento de las características del profesorado, pues ello se convierte en un elemento clave para el diseño y la aplicación de estrategias que permitan dar respuesta a las necesidades y potencialidades de los estudiantes, que es el punto de partida para la atención a la diversidad y el logro de la inclusión educativa.

Conviene comenzar significando que se trata de un colectivo docente con un promedio de edad de 36 años, con una experiencia promedio en la docencia de 8,4 años, de los cuales 2,7 se corresponden con el ejercicio de su actividad docente en el Instituto de Formación.

De los 29 docentes encuestados, que representan el 80,5\% del claustro, 6 (20,6\%) afirmaron no haber recibido capacitaciones pedagógicas y didácticas en los últimos cinco años. De ellos, 3 tuvieron la posibilidad de asistir a su primera capacitación de este tipo y de tener, en ese marco, su contacto inicial con contenidos referidos a la atención a la diversidad, lo que precisamente fue señalado por otros 16 docentes $(55,17 \%)$ como una de sus principales necesidades de capacitación.

La observación de las actividades y dinámicas desarrolladas por los docentes, incluidas las relacionadas con su labor como tutores de las actividades y proyectos de vinculación, permitió un acercamiento más directo a sus modos de actuación, caracterizados por su sensibilidad humana, su compromiso y disposición para la colaboración, insoslayables premisas para una mejor atención de los requerimientos de la diversidad del estudiantado.

En ese sentido, la aplicación del método biográfico narrativo aportó un valioso material, pues las narraciones de estudiantes y graduados con capacidades especiales revelaron el valor de las premisas antes señaladas, llamando además, la atención sobre los siguientes aspectos:

- El papel de las principales autoridades de la institución y de su liderazgo en relación con la inclusión educativa. 
- El compromiso de los colaboradores del Instituto con la inclusión y su disposición para contribuir al logro de sus objetivos.

- La importancia de la generación de un clima y ambiente institucional de confianza, seguridad y respeto.

- El reconocimiento del aporte de las becas y ayudas económicas en la continuidad de estudios de los estudiantes con discapacidad y de aquellos que proceden de entornos socioeconómicos que requieren una atención priorizada.

- La necesidad de intensificar el aprovechamiento de las potencialidades de las tecnologías de la información y de las comunicaciones en el aprendizaje de la diversidad del estudiantado y como recurso para la promoción de la inclusión.

- El deseo de los estudiantes y graduados con alguna capacidad especial de tener una participación más activa en tareas de investigación que aborden la problemática de la discapacidad y su interés en que puedan ser tenidas en cuenta sus opiniones y experiencias en las publicaciones que en relación con este tema se realicen.

- La positiva valoración que hacen los estudiantes y graduados con discapacidad de las actividades y proyectos de vinculación que en su opinión les han ayudado a desenvolverse mejor, a sentirse útiles y a contar con un mejor conocimiento de realidades que a veces les resultan distantes.

Los hallazgos obtenidos mediante la aplicación de diferentes métodos apuntan claramente hacia la verificación de la hipótesis general de la investigación, generando al mismo tiempo, pautas, reflexiones y sugerencias que podrán ser utilizadas como estímulo para el desarrollo de futuras líneas de investigación en una temática de indiscutible actualidad y trascendencia para la Educación Superior en el Ecuador y a nivel internacional.

\section{Conclusiones}

Los resultados de esta investigación permiten el análisis y la ejemplificación de algunas de las principales implicaciones que la inclusión educativa tiene para la docencia, la investigación, la vinculación y la gestión, es decir, los procesos sustantivos en torno a los cuales se articula la labor de las Instituciones de Educación Superior (IES).

Parece no admitir discusión el hecho de que el principal apoyo del que disponen las IES para superar las barreras que obstaculizan la inclusión educativa son sus docentes, quienes, al contar con la preparación requerida, que se favorece por el establecimiento de un sistema de acompañamiento metodológico, y a partir de su compromiso, sensibilidad humana y entrega, están en capacidad de garantizar una respuesta educativa de calidad para la diversidad de todo el estudiantado.

La labor del profesorado debe tomar como uno de sus criterios iniciales la consideración del nivel de partida de los estudiantes como base para la promoción de su participación protagónica y la estimulación de las interacciones que en las condiciones del aula se generan, propiciando su transformación en una comunidad de apoyo (González-Gil, 2009) que beneficia el aprendizaje de todos y cada uno de los miembros del grupo. 
A su vez, son múltiples las posibilidades de la inclusión educativa para contribuir a la dinamización y al enriquecimiento de la investigación en la Educación Superior, que al garantizar la participación de los estudiantes con discapacidad en su desarrollo crea un espacio para que su voz incorpore su auténtico timbre con intensidad propia a un coro que, sin la misma, continuaría realizando una interpretación inacabada.

Igualmente son amplias las posibilidades de la inclusión educativa en relación con la vinculación que adquiere, gracias a este proceso, un carácter doblemente inclusivo que refuerza la imprescindible vocación y compromiso social de la Educación Superior. Del mismo modo en la gestión de las IES también se evidencian las posibilidades transformadoras de la inclusión educativa, que están indisolublemente ligadas al papel de los líderes y a su decisiva contribución en la elevación del nivel de motivación, conciencia y compromiso de sus colectivos.

Todo lo antes señalado aporta evidencias teóricas y empíricas que revelan algunas de las contribuciones mediante las cuales la inclusión educativa potencia el desarrollo de los procesos sustantivos de la Educación Superior, ratificando, a la vez, la alta capacidad del enfoque inclusivo para promover la innovación, el aprendizaje y la elevación continua de la calidad de la educación superior.

\section{Referencias}

Ainscow, M. (2004). El desarrollo de sistemas educativos inclusivos: ¿Cuáles son las palancas de cambio. Journal of Educational Change, 5(4), 1-20.

Alcántar, V. M. y Arcos, J. L. (2004). La vinculación como instrumento de imagen y posicionamiento de las instituciones de educación superior. Revista Electrónica de Investigación Educativa, 6(1), 1-12.

Bolívar, A. (2002). ¿De nobis ipsis silemus? Epistemología de la investigación biográficonarrativa en educación. Revista Electrónica de Investigación Educativa, 4(1), 41-66.

Botero, C. (2009). Cinco tendencias de la gestión educativa. Revista Iberoamericana de Educación, $49(2), 2-23$.

Cerbin, W. y Kopp, B. (2006). Lesson study as a model for building pedagogical knowledge and improving teaching. International Journal of Teaching and Learning in Higher Education, $18(3), 250-257$.

CES. (2013). Ley orgánica de educación superior. Quito: CES

Del Castillo, M., Fanega, S., Fernández, A., Martín, P. y Vaz, J. (2013). Innovación empresarial. Madrid: FOREM.

Echeita, G. y Ainscow, M. (2011). La educación inclusiva como derecho: marco de referencia y pautas de acción para el desarrollo de una revolución pendiente. Tejuelo: Revista de Didáctica de la Lengua y la Literatura, 12, 26-46.

Fainholc, B. (2006). Rasgos de las universidades y de las organizaciones de educación superior para una sociedad del conocimiento, según la gestión del conocimiento. Revista de Universidad y Sociedad del Conocimiento, 3(1), 34-56.

García, M. y Cotrina, M. (2012). La contribución de la universidad al desarrollo de prácticas inclusivas: dilemas y propuestas para avanzar compartiendo. Revista de Educación Inclusiva, 5(1), 123-138. 
González-Gil, F. (2009). Formación del profesorado y apoyos. En M. P. Sarto y M. E. Venegas (Coords.), Aspectos clave de la inclusión educativa (pp. 143-158). Salamanca: INICO

Herdoiza, M. (2015). Construyendo igualdad en la educación superior. Fundamentación y lineamientos para transversalizar los ejes de igualdad y ambiente. Quito: Senescyt/Unesco.

López Melero, M. (2011). Barreras que impiden la escuela inclusiva y algunas estrategias para construir una escuela sin exclusiones. Innovación Educativa, 21, 37-54.

Machucho, F. T. y López, T. G. (2012). Vinculación, imagen y posicionamiento de una IES en la región de San Andrés Tuxtla, Veracruz. Ciencia Administrativa, 2, 11-24.

Malagón, L. A. (2006). La vinculación universidad-sociedad desde una perspectiva social. Educación y Educadores, 9(2), 79-93.

Michavila, F. (2009). La innovación educativa. Oportunidades y barreras. Arbor, 185, 3-8. doi: 10.3989/arbor.2009.extran 1201

Montenegro, H. y Fuentealba, R. (2010). El formador de futuros profesionales: una nueva forma de comprender la docencia en la Educación Superior Universitaria. Calidad en la Educación, 32, 253-267.

Salazar, M. (2006). El liderazgo transformacional: ¿modelo para organizaciones educativas que aprenden. UNIrevista, 1(3), 1-12.

Sañudo, L. (2005). La transformación de la gestión educativa. Madrid: CEDP. Recuperado de https://scholar.googleusercontent.com/scholar?q=cache:NVoWsly 1jNEJ:scholar.google. com/+La+transformación+de+la+gestión+educativa.+\&hl=es\&as_sdt=0,5Educar

Susinos, T. S. y Rodríguez-Hoyos, C. (2011). La educación inclusiva hoy: Reconocer al otro y crear comunidad a través del diálogo y la participación. Revista Interuniversitaria de Formación del Profesorado, 70, 15-30.

Talízina, N. (1993). Los fundamentos de la enseñanza en la educación superior. México: Ángeles Editores.

Tristá, B. y Álvarez Vázquez, Y. (2010). El trabajo metodológico en la educación superior. Un enfoque desde la gestión del conocimiento y el aprendizaje organizacional. Pedagogía Universitaria, 15(4), 67-77.

UNESCO. (1998). La educación superior en el siglo XXI. Visión y acción. París: UNESCO.

\section{Breve CV del autor}

\section{Rafael Félix Bell Rodríguez}

Graduado de Educación Especial con especialización en Sordopedagogía (Moscú, 1985). Máster of Arts en Educación y Máster en Educación Especial. Doctor en Ciencias Pedagógicas por el Instituto Central de Ciencias Pedagógicas, La Habana, Cuba (2009). Docente universitario e investigador certificado por la Academia de Ciencias de Cuba. Ha promovido y participado como Presidente de comisión, conferencista magistral y ponente en numerosos eventos científicos nacionales e internacionales. Autor y coautor de más de 50 publicaciones, entre las que se incluyen libros, capítulos de libros, traducciones, ponencias y artículos científicos. Actualmente se desempeña como Vicerrector académico del Instituto Tecnológico Superior de Formación Profesional, 
R. F. Bell

Administrativa y Comercial de Guayaquil, Ecuador. ORCID ID: 0000-0002-0255-642X.

Email: rafael.bell@formacion.edu.ec 